\title{
Structural Regularity of Poly(phenylsilsesquioxane) Prepared from the Low Molecular Weight Hydrolysates of Trichlorophenylsilane
}

\author{
Eung-Chan LeE and Yoshiharu Kimura \\ Department of Polymer Science and Engineering, Kyoto Institute of Technology, \\ Matsugasaki, Sakyo-ku, Kyoto 606, Japan
}

(Received August 21, 1997)

\begin{abstract}
Various hydrolysates of trichlorophenylsilane (TCP) and their polycondensates were reacted with trichlorosilane for capping the silanol groups formed in defect points of the ladder structure. The ${ }^{1} \mathrm{H}$ NMR spectra of the capping products obtained showed several signals due to the $\mathrm{Me}_{3} \mathrm{Si}$ groups in different environment. Particularly, the polycondensate prepared from the TCP hydrolysate having higher molecular weight showed both sharp and broad $\mathrm{Me}_{3} \mathrm{Si}$ signals, suggesting a core-shell type branched structure. No $\mathrm{Me}_{3} \mathrm{Si}$ signal was shown in the polycondensate prepared from the hydrolysate with low molecular weight, supporting an almost perfect ladder structure with few bonding defects. The wide angle X-ray scattering (WAXS) of this polycondensate showed clear Debye-Scherrer rings due to the crystallization of the polymer chains having the regular ladder structure. The structural regularity of this polycondensate was also supported by the ${ }^{29} \mathrm{Si}$ NMR spectrum. This polymer, having a stable double-strand structure, was found to undergo no base-catalyzed depolymerization in solution. A stepwise polycondensation is proposed for the formation of the ladder polymer in which the trivalent $\left(\mathrm{T}_{0}: \mathrm{PhSi}\left(\mathrm{O}^{-}\right)_{3}\right)$ or divalent $\left(\mathrm{T}_{1}\right.$ : $\left.\mathrm{PhSi}(\mathrm{OSi})\left(\mathrm{O}^{-}\right)_{2}\right)$ siloxyl units should be involved in the reaction, leaving the monovalent siloxyl $\left(\mathrm{T}_{2}\right.$ : $\left.\mathrm{PhSi}(\mathrm{OSi})_{2}\left(\mathrm{O}^{-}\right)\right)$units unreacted.
\end{abstract}

KEY WORDS Hydrolysate of Trichlorophenylsilane / Poly(phenylsilsesquioxane) / Silanol Capping / Ladder Structure / Core-Shell Type Branched Structure / Depolymerization /

It was found in the 1870 s that the alkaline treatment of the condensation products of phenylsilanetriol (PST) produces soluble oligomers having the empirical composition of $\left(\mathrm{C}_{6} \mathrm{H}_{5} \mathrm{SiO}_{1.5}\right)_{x}{ }^{1,2}$ Around 1960 a crystalline polycyclic compound with identical composition was isolated from a solution of the hydrolysis product of trichlorophenylsilane (TCP) and $\mathrm{KOH}$ in diethyl ether/benzene mixture. ${ }^{3,4}$ In 1963 a soluble poly(phenylsilsesquioxane) (PPSQ) with very high molecular weight was prepared by the base-catalyzed bulk polycondensation of the hydrolysis product of TCP in bulk at high temperature. ${ }^{5,6}$ This double-chain ladder polymer may be formed in equilibrium with low molecular weight polycyclic species that could be isolated from a dilute solution system. ${ }^{7}$ The reaction mechanism of these interesting condensation systems, however, has been controversial, and the products with defined structure and properties could be hardly obtained in many experiments previously described in the literatures. ${ }^{5-11}$ Recently, ${ }^{12}$ we succeeded in isolating the low molecular weight hydrolysates from the aqueous layer by hydrolyzing TCP in a water/toluene solvent system and found that the base-catalyzed polycondensation of these hydrolysates affords a high molecular weight PPSQ under mild reaction conditions. Based on these facts we proposed a new mechanistic explanation for the formation of ladder structure in the dehydrative polycondensation of the TCP hydrolysates. This paper describes the further extension of our study on the structure and formation mechanism of PPSQ by focusing on the structure regularity of the crystalline PPSQ formed from the low-molecular weight hydrolysates. In the present study, the structure of PPSQ was analyzed by capping reactions of the silanol groups with chlorotrimethylsilane (TMC) (Scheme 1) as well as by spectroscopic and $\mathrm{X}$-ray analyses. The PPSQ formed from PST and its early condensates should have highly regular structure without bonding defects.

\section{EXPERIMENTAL}

Materials

TCP and TMC were supplied by Nacalai Tesque Inc. (Kyoto) and purified by distillation. Toluene and cyclohexane were distilled over sodium metal before use. Other solvents and reagents were commercially supplied and used without purification.

\section{Measurements}

${ }^{29} \mathrm{Si} \mathrm{NMR}$ spectra were measured at $99.3 \mathrm{MHz}$ on

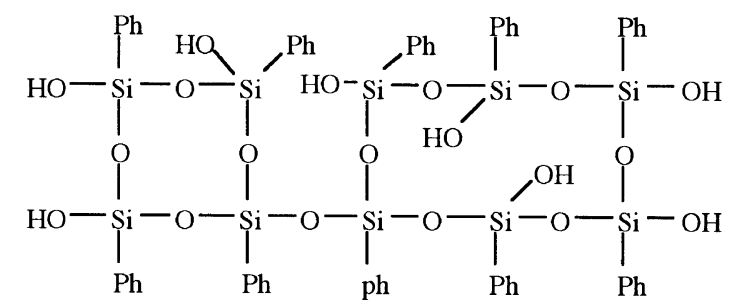
TMC

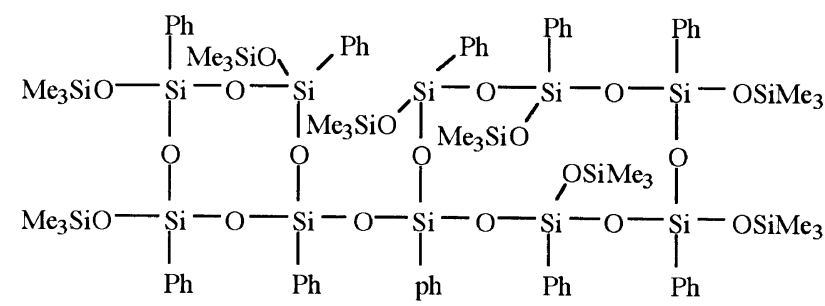

Scheme 1. 
a Brucker ARX-500 spectrometer in chloroform- $d_{1}$ $\left(\mathrm{CDCl}_{3}\right)$ and toluene- $d_{8}$. A capillary glass tube containing pure tetramethylsilane (TMS) as the in situ external standard was put into an NMR sample tube made of glass $(5 \mathrm{~mm} \phi)$ together with a sample solution. Measurement was done at $21^{\circ} \mathrm{C}$ by spinning the sample tube at a revolution of $5000 \mathrm{~s}^{-1} \cdot{ }^{1} \mathrm{H}$ NMR spectra were measured at $200 \mathrm{MHz}$ on a Varian XL-200 spectrometer in $\mathrm{CDCl}_{3}$ with the solvent peak as the internal reference $(\delta$ 7.26). The number average molecular weight $\left(M_{n}\right)$ and molecular weight distribution $\left(M_{w} / M_{n}\right)$ were determined by gel permeation chromatography (GPC). The analyzer was composed of a Shimadzu LC-10A pump, a Shodex RI SE-31 RI detector, and a Shimadzu C-R7A Chromatopac data processor. A combination of two polystyrene gel columns of Tosoh TSK gel G4000 $\mathrm{H}_{8}$ and $\mathrm{G} 2500 \mathrm{H}_{8}$ was used with chloroform as the eluent at $35^{\circ} \mathrm{C}$. The limited exclusion molecular weight was $7 \times 10^{5}$ dalton. The molecular weight was calibrated according to polystyrene standards. FT-IR spectra were recorded on a JASCO FT/IR-5300 spectrophotometer by the $\mathrm{KBr}$ pellet method. Wide angle X-ray scattering (WAXS) was measured on a JEOL DX-GE-E model diffractometer with $\mathrm{Ni}$-filtered $\mathrm{Cu}-K_{\alpha}$ radiation at a wave length of $1.542 \AA$ generated at $40 \mathrm{kV}$ and $20 \mathrm{~mA}$. Density was measured by the floating method using a heavy salt solution of $\mathrm{KI} /$ water.

\section{Preparation of TCP Hydrolysates}

The procedure was as reported previously. ${ }^{12} \mathrm{~A}$ solution of $6.3 \mathrm{~g}$ of TCP in $163 \mathrm{ml}$ of toluene was cooled to $0^{\circ} \mathrm{C}$ and added dropwise to $815 \mathrm{ml}$ of water under vigorous agitation at $0^{\circ} \mathrm{C}$ for a period of $1 \mathrm{~h}$. The mixture was kept stirred at the same temperature for $30 \mathrm{~min}$, and the aqueous and toluene layers were separated at $0^{\circ} \mathrm{C}$. The $\mathrm{pH}$ of the aqueous layer was adjusted to 4 by adding aqueous $\mathrm{NaHCO}_{3}$ solution containing some ice to allow precipitation of the white crystalline product whose $M_{n}$ was about 170 dalton. The white precipitates were filtered and dried throughly in vacuo at room temperature; yield: $0.50 \mathrm{~g}(9.3 \%)$. The filtrate was kept in a refrigerator at $0^{\circ} \mathrm{C}$ overnight for precipitation of the hydrolysate having $M_{n}=290$. The precipitated product was isolated by filtration, washed with an ice-water, and dried in vacuo; yield: $5.04 \mathrm{~g}(80 \%)$.

When the same hydrolysis reaction was carried out without cooling the toluene solution of TCP, no crystalline hydrolysate having $M_{n}=170$ was obtained from the aqueous solution whose $\mathrm{pH}$ had been adjusted at 4 . The $\mathrm{pH}$-adjusted solution was kept at $0^{\circ} \mathrm{C}$ overnight, the hydrolysate having $M_{n}=540$ precipitated out as a solid. Which was isolated and dried as above; yield: $5.04 \mathrm{~g}$ $(80 \%)$.

In another experiment, the same TCP solution in toluene was added dropwise to the water layer at room temperature, and the toluene and aqueous layers were separated. In this case, the toluene layer was evaporated to dryness at reduced pressure to obtain the hydrolysate having $M_{n}=1560$ in a yiled of $5.3 \mathrm{~g}(84 \%)$.

\section{Polycondensation}

$5.0 \mathrm{~g}$ of the TCP hydrolysate and $5.0 \mathrm{mg}$ of $\mathrm{KOH}$ $\left(0.1 \mathrm{wt}^{\%} \%\right.$ relative to the hydrolysate) were mixed with
$38 \mathrm{ml}$ of toluene. The mixture was heated at the refluxing temperature of toluene for $16 \mathrm{~h}$ with the dehydrated water removed from the azeotropically distilled toluene/water mixture in a Dean-Stark tube. The viscous solution finally obtained was poured into excess methanol to precipitate the condensation product. The product was then filtered and dried in vacuo at $150^{\circ} \mathrm{C}$ for $10 \mathrm{~h}$; yield: $4.4 \mathrm{~g}(88 \%)$. It was dissolved in toluene, cast on a glass plate, and air-dried to prepare a polymer film. The halfdried film was then taken off and thoughly dried in a vacuum oven at $150^{\circ} \mathrm{C}$. A fragment of this film was heattreated at $350^{\circ} \mathrm{C}$ in soild state.

\section{Silanol-Capping Reaction}

A typical reaction was carried out as follows: $3.3 \mathrm{~g}$ of the TCP hydrolysate $\left(M_{n}=540\right)$ obtained above were mixed with $4.4 \mathrm{~g}(43 \mathrm{mmol})$ of triethylamine in $100 \mathrm{ml}$ of cyclohexane. With this solution stirred, $4.7 \mathrm{~g}(43 \mathrm{mmol})$ of TMC dissolved in $100 \mathrm{ml}$ of cyclohexane were added dropwise below $20^{\circ} \mathrm{C}$. Stirring was continued for $90 \mathrm{~min}$. The white precipitates having formed during the reaction were removed by filtration, and the filtrate was evaporated to dryness to obtain yellowish powdery product, with a small portion of cyclohexane and thoroughly dried in vacuo. The yield of the product was $2.5 \mathrm{~g}$. Capping reactions of the other TCP hydrolysates and their polycondensates were performed likewise by reacting excess amounts of triethylamine and TMC with the reactants. The IR spectra of the capping products showed absorption bands at $3080-2940(\mathrm{C}-\mathrm{H}$ stretching), 1435 ( $\mathrm{Si}-$ phenyl groups), 1050 (O-Si-phenyl), $1150(\mathrm{O}-\mathrm{Si}), 750$ and 700 (phenyl groups) $\mathrm{cm}^{-1}$. These data were fundamentally similar to those of the corresponding TCP hydrolysate or its polycondensate except for the absorptions due to the trimethyl groups introduced.

Table I. Changes in molecular weight of polycondensates prepared from the TCP hydrolysates having different molecular weight ${ }^{\mathrm{a}}$

\begin{tabular}{ccccc}
\hline \multicolumn{2}{c}{ TCP hydrolysate } & & \multicolumn{2}{c}{ Polycondensate } \\
\cline { 1 - 1 }$M_{n} /$ dalton & $M_{w} / M_{n}$ & & $M_{n} / 10^{4}$ dalton & $M_{w} / M_{n}$ \\
\hline 170 & 1.1 & & 12.0 & 1.9 \\
290 & 1.2 & & 7.4 & 1.7 \\
540 & 1.5 & & 4.6 & 2.6 \\
1560 & 3.1 & 1.4 & 3.2 \\
\hline
\end{tabular}

${ }^{a}$ Reported in the previous paper. ${ }^{12}$

Table II. Results of end-capping reaction of TCP hydrolysates $(\mathrm{Hy})$ and polycondensates $(\mathrm{Cn})$

\begin{tabular}{|c|c|c|c|c|c|}
\hline $\begin{array}{l}\text { Run } \\
\text { No. }\end{array}$ & $\begin{array}{l}\text { Sample } \\
\left(M_{n} \text { in }\right. \\
\text { dalton })\end{array}$ & $\frac{\text { Yield }}{\%}$ & $\frac{M_{n}}{\text { dalton }}$ & $M_{w} / M_{n}$ & $\begin{array}{l}\text { Unit ratio } \\
\mathrm{Me}_{3} \mathrm{Si} / \mathrm{PhSi}\end{array}$ \\
\hline 1 & Hy (170) & 82 & 390 & 1.0 & $1.1 / 1.0$ \\
\hline 2 & Нy (290) & 82 & 730 & 2.1 & $0.56 / 1.0$ \\
\hline 3 & Hy (540) & 85 & 1,200 & 3.1 & $0.57 / 1.0$ \\
\hline 4 & Hy $(1,560)$ & 88 & 1,900 & 3.2 & $1.27 / 1.0$ \\
\hline 5 & $\operatorname{Cn}(14,000)$ & 91 & 17,000 & 3.2 & $58 / 1000$ \\
\hline 6 & $\mathrm{Cn}(46,000)$ & 89 & 52,000 & 2.6 & $3.8 / 1000$ \\
\hline 7 & $\operatorname{Cn}(74,000)$ & 88 & 78,000 & 1.7 & $2.0 / 1000$ \\
\hline 8 & Cn $(120,000)$ & 88 & 121,000 & 1.9 & $0.0 / 1000$ \\
\hline
\end{tabular}

${ }^{a}$ By ${ }^{1} \mathrm{H}$ NMR. 
a)
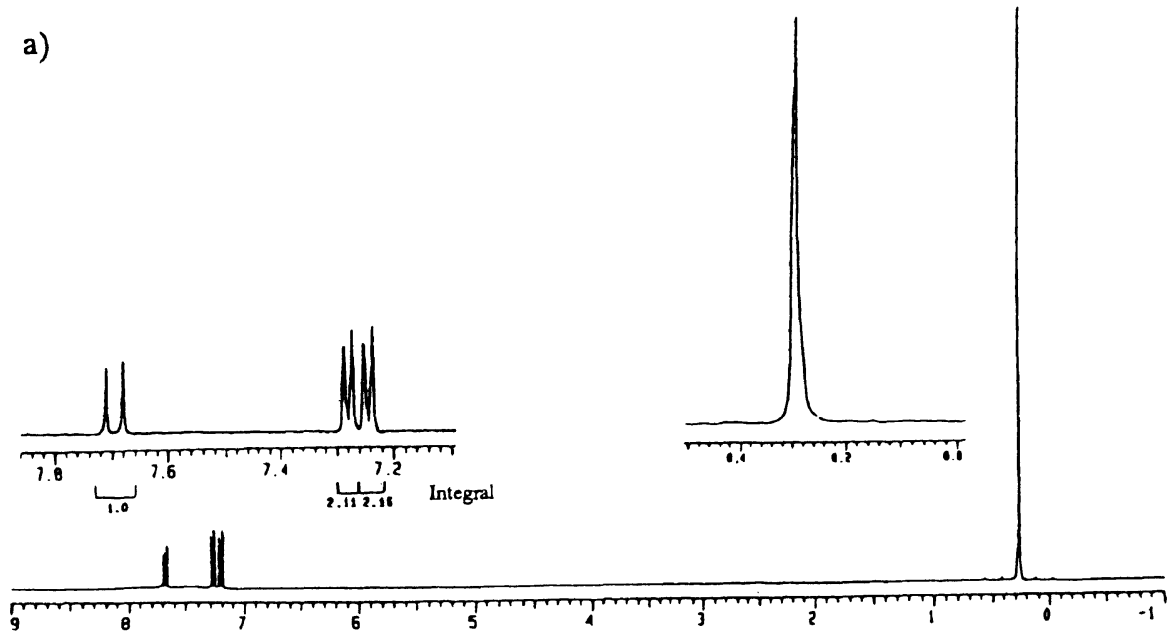

$\delta / \mathrm{ppm}$

b)
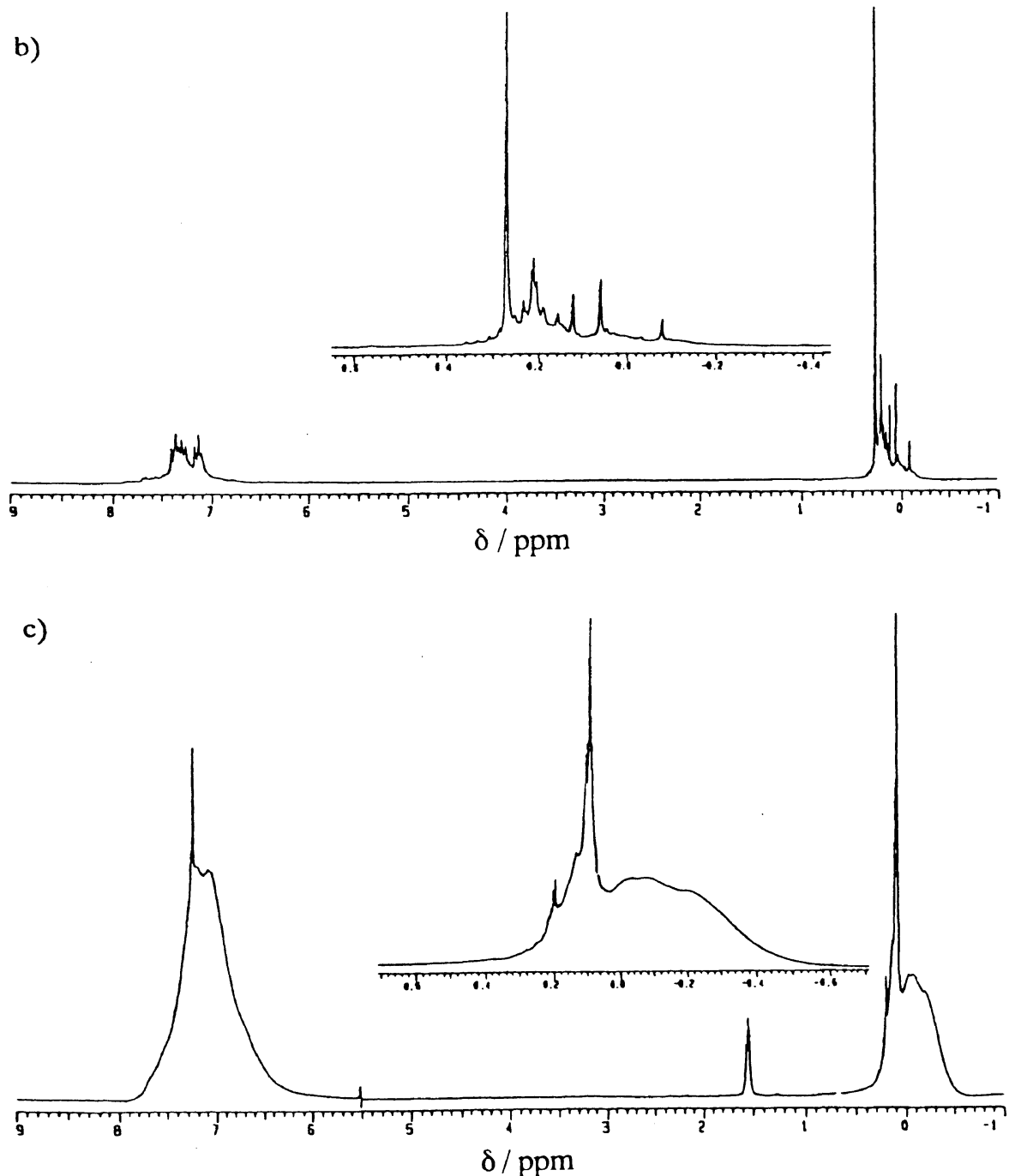

Figure 1. ${ }^{1} \mathrm{H}$ NMR spectra of the capping products obtained from the hydrolysates having $M_{n}=$ (a) 170 , (b) 290 , and (c) 1560 .

\section{Depolymerization}

$2.5 \mathrm{~g}$ of PPSQ prepared from the hydrolysate having $M_{n}=170$ were dissolved in $76 \mathrm{ml}$ of toluene. Into this solution $2.5 \mathrm{mg}$ of $\mathrm{KOH}$ were added, and the mixture was refluxed for $20 \mathrm{~h}$. The solution was poured into excess methanol to precipitate the product. The white resinous product was fitered and dried in vacuo. Recovery of the product was $2.32 \mathrm{~g}(94 \%)$.

\section{RESULTS AND DISCUSSION}

Detection of Structural Defects by Silanol Capping

The structure and molecular weight of PPSQ are greatly affected by the type of TCP hydrolysate used in 


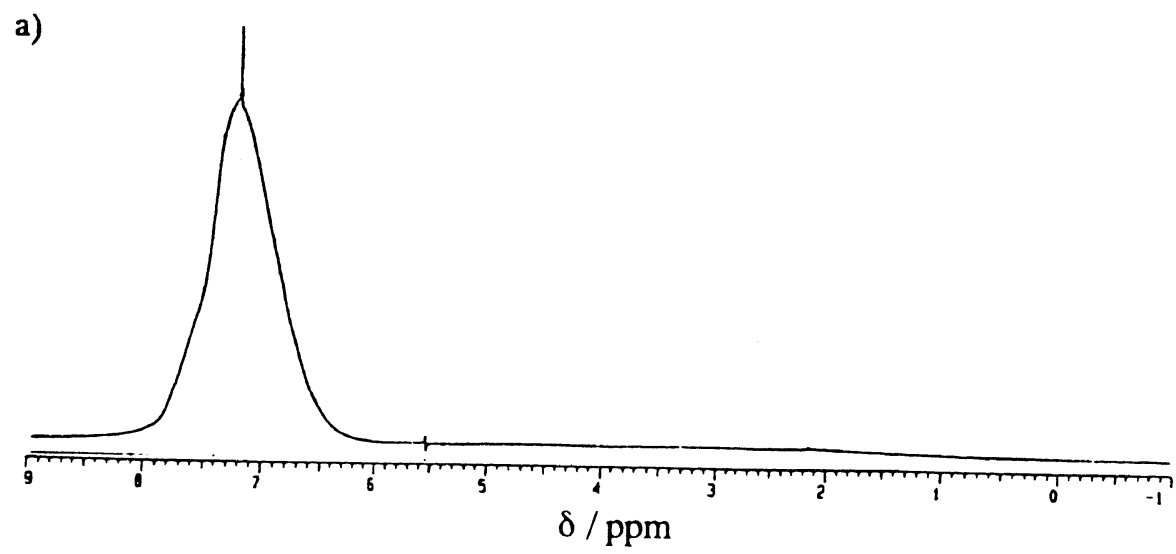

b)

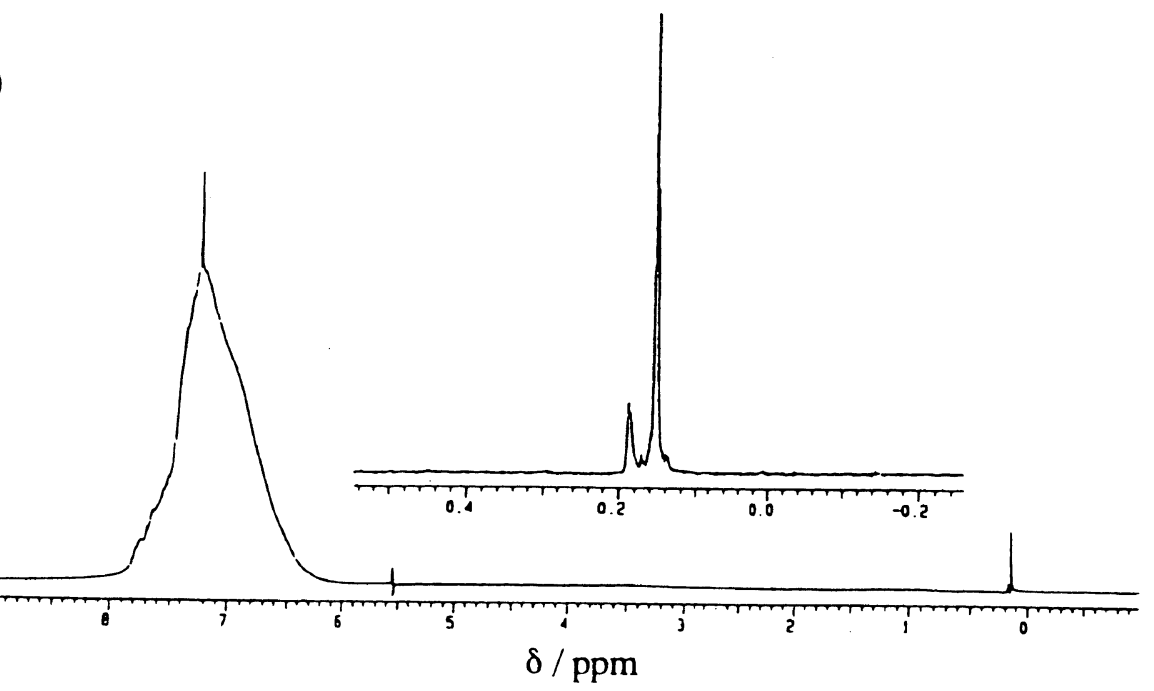

c)

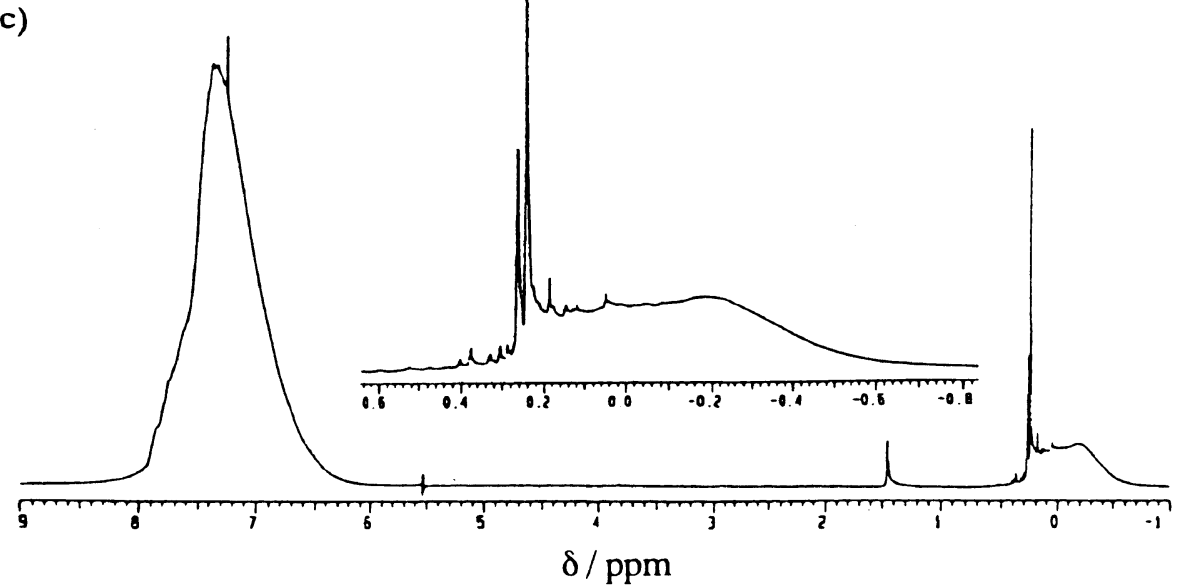

Figure 2. ${ }^{1} \mathrm{H}$ NMR spectra of the capping products obtained from the polycondensates having $M_{n}=$ (a) 120000 , (b) 74000 , and (c) 14000 .

the $\mathrm{KOH}$-catalyzed polycondensation. A typical relationship between the molecular weight of the hydrolysate and that of the polycondensate is illustrated in Table I whose data were reported in the previous paper. ${ }^{12}$ It is clearly shown that the degree of polymerization of PPSQ increases with decreasing molecular weight of the starting hydrolysate. IR spectroscopy suggested that a double-stranded structure of PPSQ should become more regular with decreasing molecular weight of the hydrolysate. Particularly, the high-molecular weight PPSQ prepared from the hydrolysate consisting mainly of PST (monomeric hydrolysate) seemed to consist of a completely regular ladder sequence. The regularity of the double-stranded structure of PPSQ has not been well 

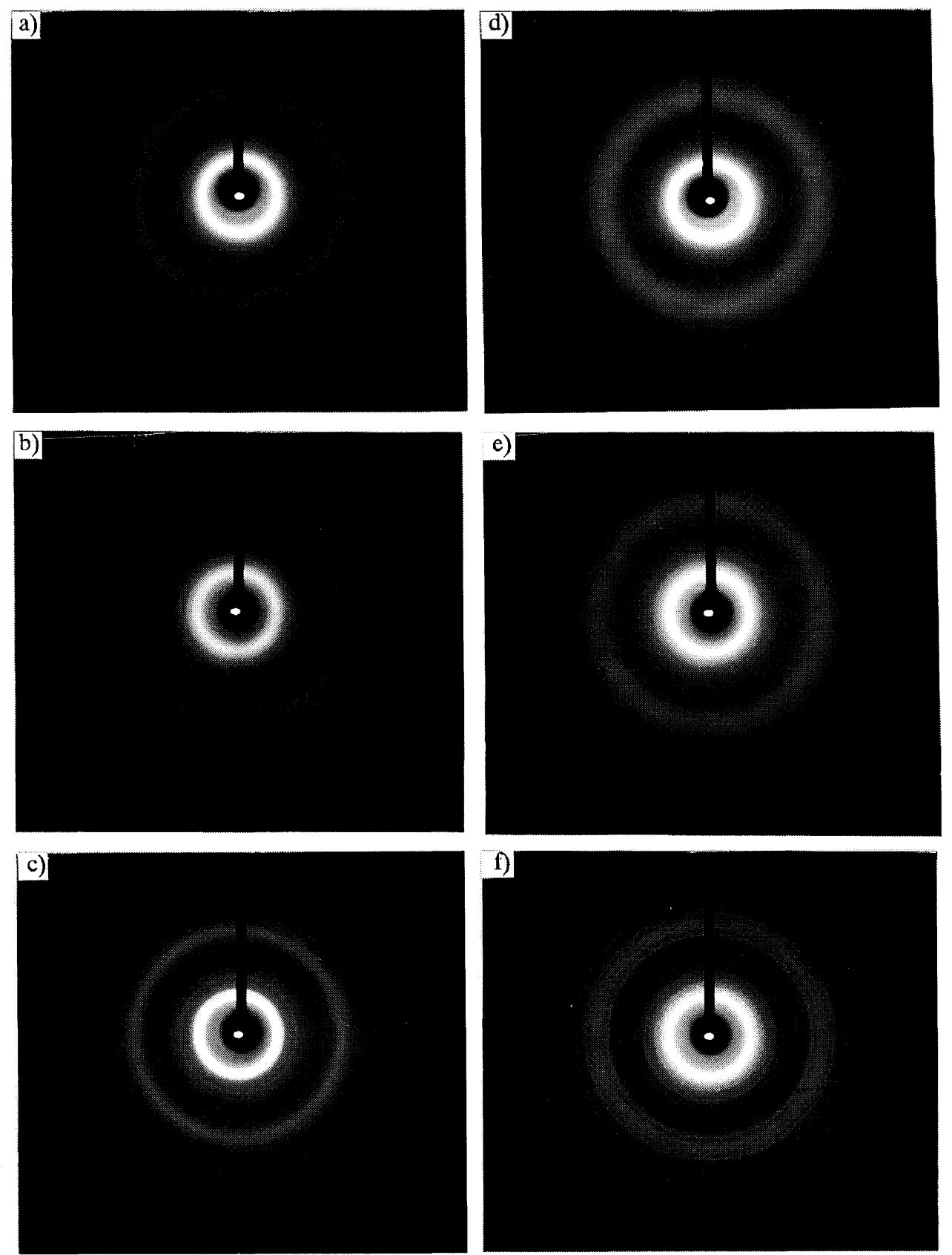

Figure 3. WAXS of the as-prepared (AP) and heat-treated (HT) PPSQ films; (a) $M_{n}=14000$, AP; (b) $M_{n}=74000$, AP; (c) $M_{n}=120000$, AP; (d) $M_{n}=14000$, HT; (e) $M_{n}=74,000$, HT; (f) $M_{n}=120000$, HT.

identified by other analytical methods, and it is controversial how many structural defects would be contained in the ladder sequence ${ }^{13,14}$ or at what stage of the polycondensation the ladder structure would be formed. ${ }^{15,16}$

Here, the silanol groups of the TCP hydrolysates and their polycondensates were trimethylsilylated to cap and detect them by ${ }^{1} \mathrm{H}$ NMR spectroscopy. Analysis of the introduced silanol groups should give insight into the structures of the polymer tails and the defect points of the ladder structure. Table II summarizes the results of the silanol capping reactions for various TCP hydrolysates and their polycondensates. In each case, recovery of the capping product was higher than $80 \%$. The molecular weights of the capping products became significantly higher than the original compounds probably because some of the labile silanol groups had incurred intermolecular coupling during the capping reaction. Therefore, this method could not provide the quantitative analysis of the silanol groups in spite of much important structural information.

The typical ${ }^{1} \mathrm{H}$ NMR spectra of the capping products of the TCP hydrolysates and their polycondensates are shown in Figures 1 and 2, respectively. It is known that most capping products show ${ }^{1} \mathrm{H}$ signals around $7-8 \mathrm{ppm}$ and $-0.5-0.3 \mathrm{ppm}$ which are attributed to the $\mathrm{PhSi}$ and $\mathrm{Me}_{3} \mathrm{Si}$ groups, respectively. From the integral ratio of these signals, the unit ratio of $\mathrm{Me}_{3} \mathrm{Si} / \mathrm{PhSi}$ in each capping product was calculated. The results are summarized in the last column of Table II. For the capping product from the hydrolysate having $M_{n}=170$ (Figure 1a), which showed only one GPC peak, the unit ratio of $\mathrm{Me}_{3} \mathrm{Si} / \mathrm{PhSi}$ 
Table III. Results of X-ray diffraction of the PPSQ $\left(M_{n}=120000\right)$ film heat-treated at $350^{\circ} \mathrm{C}$

\begin{tabular}{|c|c|c|c|c|c|}
\hline Debye- & $2 \theta$ & $d$-Spacing & Debye- & $2 \theta$ & $d$-Spacing \\
\hline ring & $\circ$ & $\AA$ & ring & $\circ$ & $\AA$ \\
\hline$r_{1}$ & 8.18 & 12.01 & $r_{7}$ & 22.90 & 4.31 \\
\hline$r_{2}$ & 10.41 & 9.43 & $r_{8}$ & 23.92 & 4.13 \\
\hline$r_{3}$ & 12.12 & 8.11 & $r_{9}$ & 25.92 & 3.81 \\
\hline$r_{4}$ & 13.24 & 7.43 & $r_{10}$ & 27.86 & 3.55 \\
\hline$r_{5}$ & 19.78 & 4.93 & $r_{11}$ & 29.75 & 3.33 \\
\hline$r_{6}$ & 21.87 & 4.51 & $r_{12}$ & 31.58 & 3.14 \\
\hline
\end{tabular}
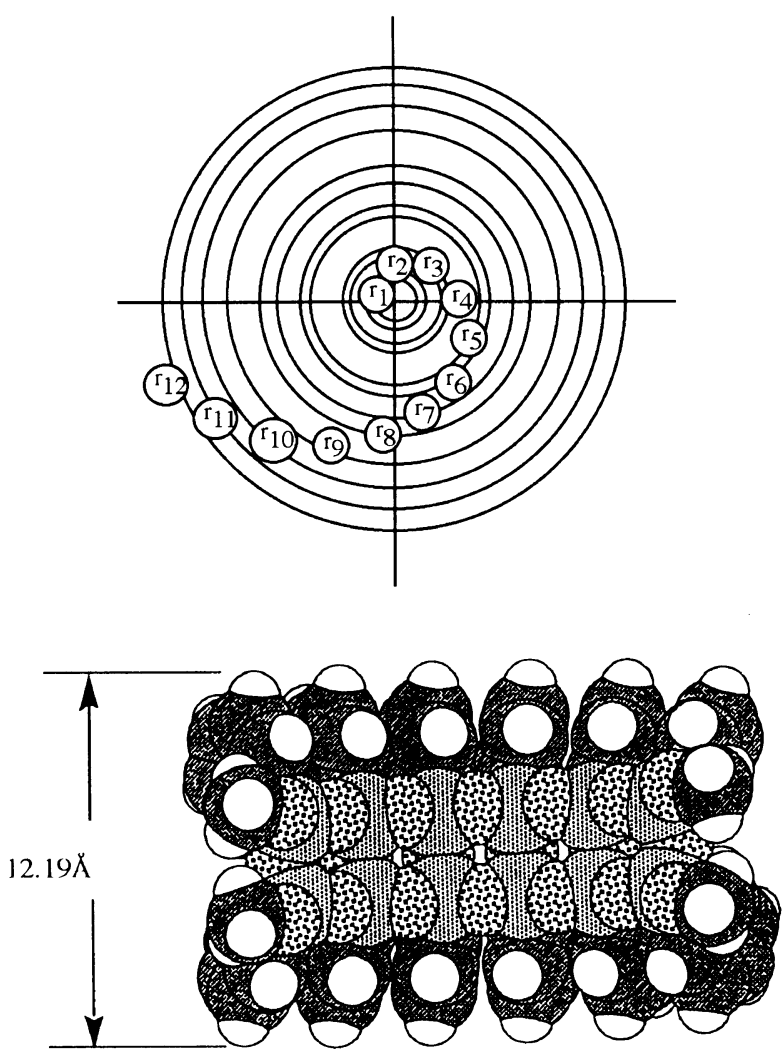

cis-isotactic

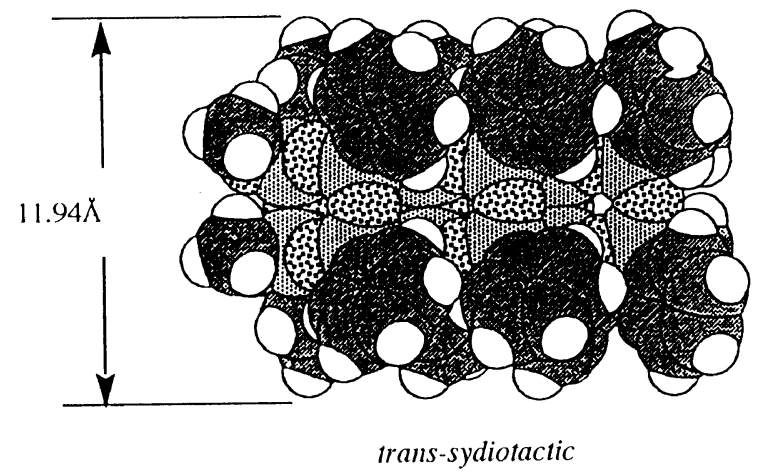

Scheme 2.

was $1.1 / 1.0$ and much lower than the ratios of $3 / 1$ and $2 / 1$ for the ideal capping products of PST (monomeric) and dimeric hydrolysate of $\left[\mathrm{PhSi}(\mathrm{OH})_{2}\right]_{2} \mathrm{O}$, respectively. Observation of the sole $\mathrm{Me}_{3} \mathrm{Si}$ signal, indicated that this product should have a simple cyclic structure which probably might have been produced both through

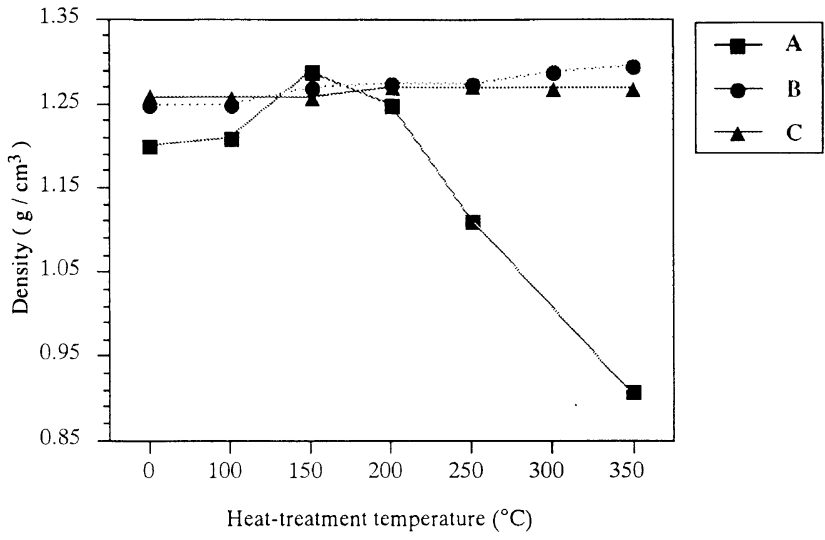

Figure 4. Temperature-dependent changes in density of the PPSQ films having $M_{n}=120000$ (A), (b) 74000 (O), and (c) 14000 ( $\mathbf{\square}$ ).

intermolecular coupling and intramolecular cyclization during the capping reaction. Therefore, the original hydrolysate itself should have such a simpler structure as PST monomer or dimer. ${ }^{17,18}$ The capping product from the hydrolysate having $M_{n}=290$ showed several small signals due to the $\mathrm{Me}_{3} \mathrm{Si}$ groups around $-0.1-$ $0.3 \mathrm{ppm}$ in addition to the large singlet signal (Figure 1b). These high-field signals were mainly separated into five peaks and became larger in the capping product from the hydrolysate having $M_{n}=540$. Therfore, these capping products would have a complex branched structure in which the $\mathrm{Me}_{3} \mathrm{Si}$ groups exist in different environment. The capping product of the hydrolysate having $M_{n}=$ 1560 , isolated from the toluene layer, showed sharp and broad signals around $0 \mathrm{ppm}$ together with a broad phenyl signal (Figure 1c). The former sharp and broad signals should probably be attributed to the $\mathrm{Me}_{3} \mathrm{Si}$ groups with high and restricted mobilities, respectively. ${ }^{13}$ This strongly suggests that tri-dimensionally branched structure of a core-shell type is formed in this hydrolysate and that the $\mathrm{Me}_{3} \mathrm{Si}$ groups in the core should have low mobility resulting in the broad line shape of the NMR signal while those in the shell should have an ordinary mobility and sharp line shape. The signal due to the unreacted silanol groups was detected at $1.6 \mathrm{ppm}$, indicating that some silanol groups in the core may not have reacted. These data strongly indicate that the original hydrolysate having higher molecular weight should have a complex branched structure whose complexity decreases with the molecular weight of the hydrolysate being smaller.

The capping products of the polycondensates also showed signals due to the $\mathrm{Me}_{3} \mathrm{Si}$ groups introduced, although their strength was much weaker than that of the corresponding hydrolysates. The product from the polycondensate having $M_{n}=14000$ (prepared from the hydrolysate having $M_{n}=1560$ ) showed sharp and broad $\mathrm{Me}_{3} \mathrm{Si}$ signals (Figure $2 \mathrm{c}$ ) as the capping product of the corresponding hydrolysate did. This suggests that the product should have the core-shell type branched structure. The capping product from the polycondensate having higher molecular weight showed only sharp $\mathrm{Me}_{3} \mathrm{Si}$ signals (Figure 2b) which correspond to the silanol groups at the defect points of the ladder as well as the polymer tails. The assignment of the two signals has not been made, but they may possibly be due to the $\mathrm{Me}_{3} \mathrm{Si}$ groups 


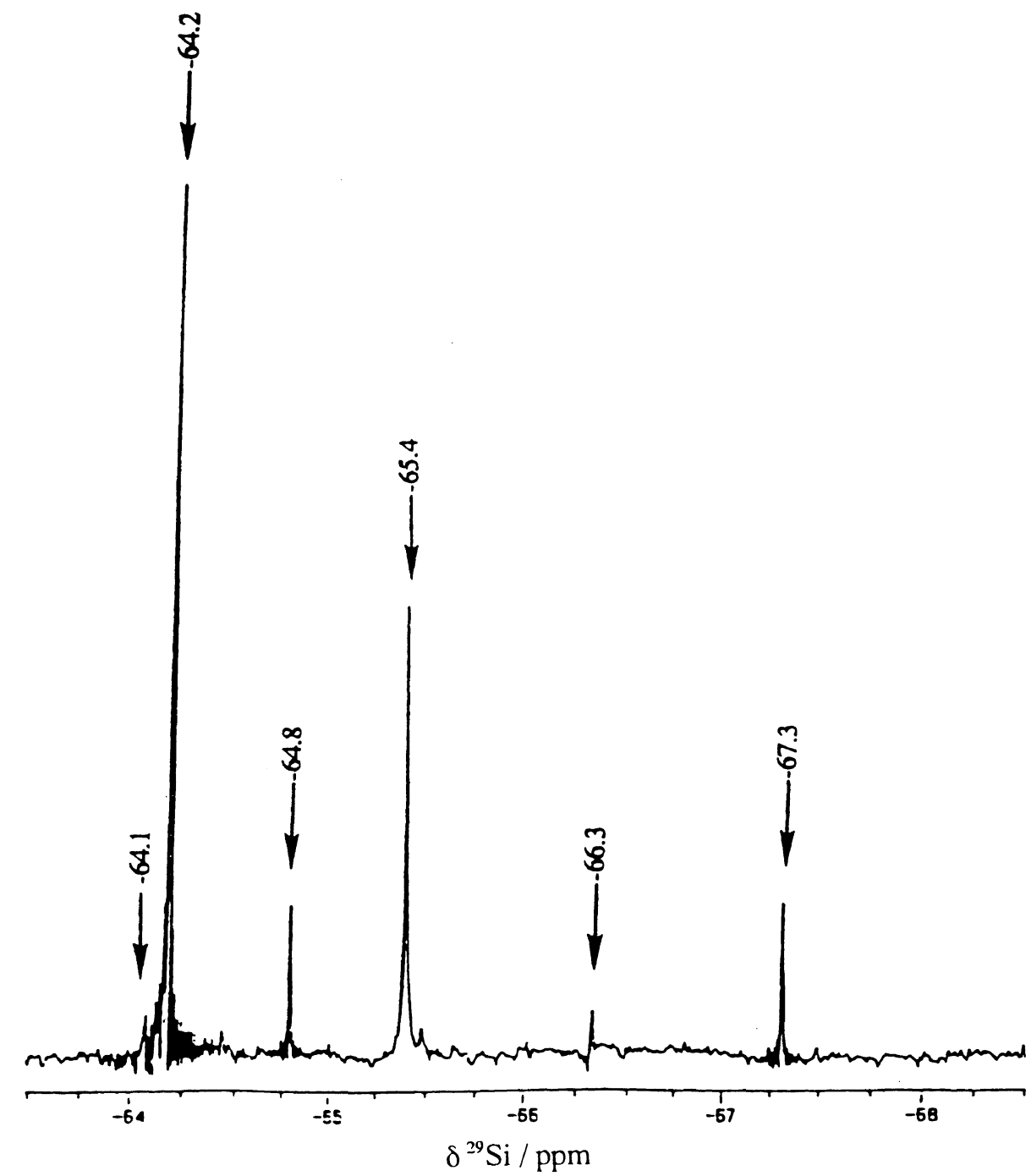

Figure 5. A typical $99.3 \mathrm{MHz}{ }^{29} \mathrm{Si}$ NMR spectrum of the hydrolysate having $M_{n}=540$ in chloroform- $d_{1}$.

in different environment which should be related to the difference in shielding effect. No $\mathrm{Me}_{3} \mathrm{Si}$ signal was detected (Figure 2a) for the product from the polycondensate having $M_{n}=120000$ (prepared from the hydrolysate having $M_{n}=170$ ), probably because it contained few structural defects in the ladder and too few end groups to be detected by NMR spectroscopy. These findings support that the PPSQ prepared from the hydrolysate consisting mainly of PST should have an almost perfect ladder structure while those from oligomeric hydrolysates should involve a significant numbers of structural defects.

\section{Solid-State Structure of PPSQ}

Figure 3 shows the WAXS of the as-prepared and heat-treated polycondensates having different molecular weight. The polycondensate having $M_{n}=14000$ showed two halos at $2 \theta=7.77^{\circ}$ and $19.08^{\circ}$ due to the partial arrangement of the repeating units. These results are almost identical with those reported by Andrianov et al., ${ }^{19}$ suggesting that PPSQ is originally crystalline. This pattern was preserved even in the heat-treated polymer whose $M_{n}$ increased to 500000 during the heat treatment in solid state. The polycondensate having $M_{n}=46000$ showed identical halos (at $2 \theta=7.98,19.52^{\circ}$ ) even after heat-treatment. The polycondensate having $M_{n}=120000$, showed many clear Debye-Scherrer rings at $2 \theta=7.96$, $20.90^{\circ}$, and $28.57^{\circ}$ which were further separated into fine ones at $2 \theta=8.18,21.87^{\circ}$, and $31.58^{\circ}$ in the heat-treated sample. The polycondensate prepared from the hydrolysate having $M_{n}=170$ is thus highly crystalline, probably because the chain structure is stereo regular. This is the first example of crystalline PPSQ whose Debye-Scherrer rings can be distinguished accurately. Evidently, the crystal structure should be complex because of the many reflections.

The planar spacings calculated from the observed Debye-Scherrer rings are summarized in Table III. The middle $d$-spacing around $12.01 \AA$ is considered to correspond to the side lengths $\left(e . g ., \mathrm{H}_{\mathrm{Ph}}-\mathrm{H}_{\mathrm{Ph}}\right)$ of the eight-membered ring of the cyclic tetramer which is the repeating segment of the ladder chain (see Scheme 2). ${ }^{19}$ The sharpness of this reflection indicates that the polymer chains consist of cyclic tetramers as repeating segments. The short $d$-spacings around $4.51 \AA$ suggest that the chain packing should be as that of ordinary crystalline polymers. Since the double-strand chains would crystallize only when their micro structure is also regular, the polymer chains of the crystalline PPSQ should have a stereoregular structure which may be either cis-isotactic 
a)

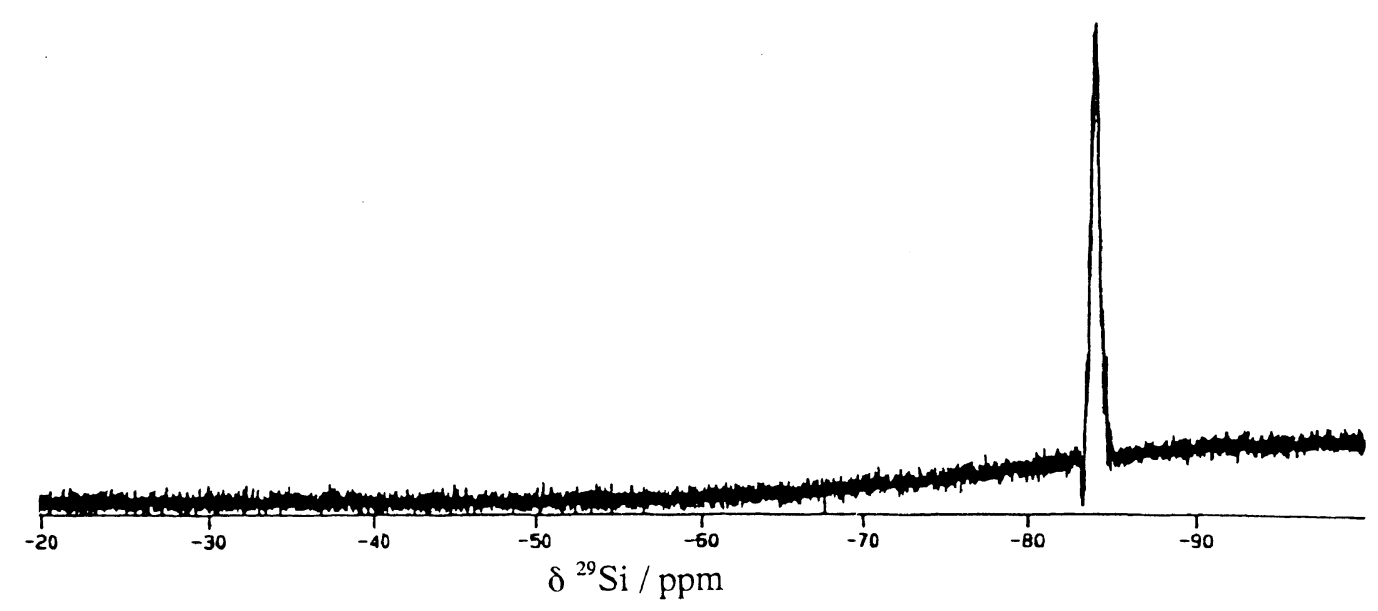

b)

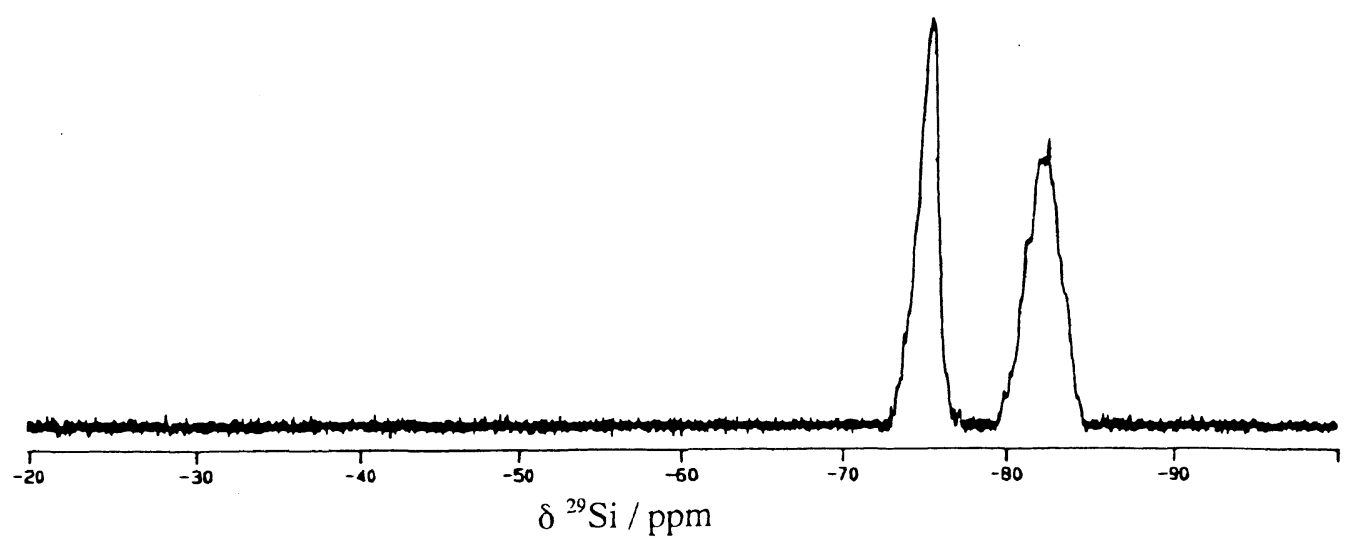

Figure 6. Typical $99.3 \mathrm{MHz}{ }^{29} \mathrm{Si}$ NMR spectra of the polycondensates having $M_{n}=$ (a) 120000 and (b) 14000 in toluene- $d_{8}$.

or trans-syndiotactic with respect to the repeating segments of the cyclic tetramer (scheme 2). ${ }^{5}$ At present the real micro structure could not be determined from the WAXS data. The preliminary molecular mechanical calculation, indicated that the trans-syndiotactic is more stable and gives a good correspondence between calculated distances and observed $d$-spacings. ${ }^{20}$ Further study will be necessary for analyzing the stereoregularity of the chain.

Figure 4 shows temperature-dependent changes in density of the polycondensates having different molecular weight. The polycondensates prepared from the hydrolysates having low $M_{n}$ showed very small changes in density, while that prepared from the hydrolysate having higher $M_{n}$ showed a large decrease above $150^{\circ} \mathrm{C}$. In the latter case the molecular weight increased with rise of temperature, and the density change should be attributed to the chain rearrangement neccessary for further polycondensation in the solid state. In the former case, little increase in molecular weight was observed with temperature. These data also support that the PPSQ prepared from the hydrolysate isolated from the aqueous layer should have regular structure that is different from the one prepared by the conventional way.

\section{Polycondensation of TCP Hydrolysate}

Figure 5 shows the $99.3 \mathrm{MHz}{ }^{29} \mathrm{Si}$ NMR spectrum of the hydrolysate having $M_{n}=540 .{ }^{21}$ To designate the structural units of the species present in the solution, the symbol $\mathrm{T}$ for the $\mathrm{PhSi}\left(\mathrm{O}^{-}\right)_{3}$ unit is used throughout this paper. The number of siloxane bridges is indicated with a numerical subscript; $\mathrm{T}_{0}: \mathrm{PhSi}\left(\mathrm{O}^{-}\right)_{3}, \mathrm{~T}_{1}: \mathrm{PhSi}(\mathrm{OSi})$ $\left(\mathrm{O}^{-}\right)_{2}, \mathrm{~T}_{2}: \mathrm{PhSi}(\mathrm{OSi})_{2}\left(\mathrm{O}^{-}\right)$, and $\mathrm{T}_{3}: \mathrm{PhSi}(\mathrm{OSi})_{3}$. In the spectrum, signals due to the monomeric PST (whose unit is depicted as $\mathrm{T}_{0}$ ) and the dimer can be detected at $\delta-64.1$ and $-64.2 \mathrm{ppm}$, respectively. The former signal was much larger in the hydrolysate having $M_{n}=170$. The signals of the trimer and tetramer are also detected as two sets of peaks with different ratios. The smaller set at -64.8 and $-66.3 \mathrm{ppm}$ should be attributed to the trimer, while the larger set at -65.4 and $-67.3 \mathrm{ppm}$ to the tetramer. In both sets the weaker signal appearing at the higher magnetic field should be attributed to the divalently connected $\mathrm{PhSi}$ unit, i.e., $\mathrm{T}_{2}$ unit, while the stronger one, to the singly connected one, i.e., $\mathrm{T}_{1}$ unit. These data support a stepwise condensation of the TCP hydrolysates in which the dimer is formed from the monomeric PST and the tetramer is formed from the dimers, although there is an additional course that the monomer and the dimer can react to produce the trimer. The hydrolysates formed in this way should be linear oligomers if the degree of condensation is low enough, and they precipitate from the aqueous layer when TCP is hydrolyzed in water/toluene bilayer system, as we reported before. ${ }^{12}$

In the $\mathrm{KOH}$-catalyzed polycondensation the trivalent 
b)

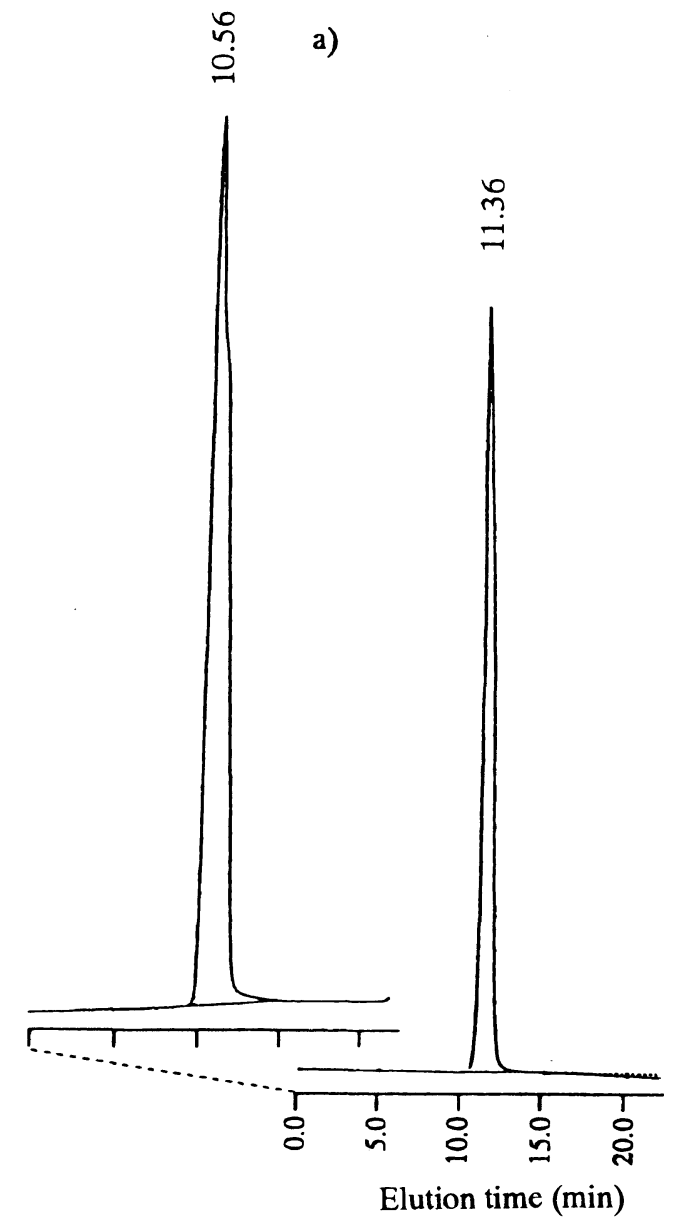

Figure 7. GPC of the PPSQ (a) before and (b) after the heat-treatment in the presence of $0.1 \mathrm{wt} \%$ of $\mathrm{KOH}$ at $110^{\circ} \mathrm{C}$ for $20 \mathrm{~h}$.

$\left(T_{0}\right)$ or divalent $\left(T_{1}\right)$ siloxyl units should be first elected to form chains, and the $T_{2}$ siloxyl units, if any, should remain somewhere in the chains. If the starting hydrolysates contain only $T_{1}$ and $T_{2}$ units, the chain extension would progress with few defects formed. If many $\mathrm{T}_{2}$ units are involved, their silanol groups would be expelled from the formation of the ladder chain and finally form the defects and chain branching. Figure 6 shows the 99.3 $\mathrm{MHz}^{29} \mathrm{Si}$ NMR spectra of the polycondensates prepared from the hydrolysates with different molecular weights. The product prepared from the low molecular weight hydrolysate $\left(M_{n}=170\right)$ showed only one signal due to the $\mathrm{T}_{3}$ units $(\delta-83 \mathrm{ppm})$, while that one from the high-molecular weight hydrolysate $\left(M_{n}=1560\right)$ showed $\mathrm{T}_{2}(\delta-75 \mathrm{ppm})$ and $\mathrm{T}_{3}(\delta-82.1 \mathrm{ppm})$ units which became broad owing to different environment in the irregular polymer sequences.

PPSQ may depolymerise into such cage compounds as octaphenylcyclotetrasiloxane (T8) when refluxed. ${ }^{22}$ Our polymer prepared from the hydrolysate having $M_{n}=170$ was dissolved in toluene and subjected to refuxing in the presence of a catalytic amount of $\mathrm{KOH}$ for $20 \mathrm{~h}$. Figure 7 shows the GPC curves of the PPSQ before and after refluxing. The molecular weight even increased after refluxing with slight increase in molecular weight distribution. This indicated that the ladder sequence is stable if the chains consist of regular double-strands. The bond cleavage and rearrangement reported before may be allowed only when the chains contain single strands.

\section{CONCLUSION}

Various TCP hydrolysates and their polycondensates were reacted with TMS for capping the silanol groups. The ${ }^{1} \mathrm{H}$ NMR spectra of the produced capping products showed several signals due to the $\mathrm{Me}_{3} \mathrm{Si}$ groups introduced. Observation of sharp and broad signals for the polycondensate prepared from the hydrolysate having $M_{n}=1560$ (isolated from the toluene layer) suggests that this polymer may have a core-shell type branched structure with many bonding defects. $\mathrm{No} \mathrm{Me}_{3} \mathrm{Si}$ signal was detected for the polycondensate prepared from the hydrolysate having $M_{n}=170$ (isolated from the aqueous layer), supporting an almost perfect ladder structure with few structural defects. WAXS analysis revealed that the latter polycondensate was highly crystalline, having a stereo regular chain structure. Its ${ }^{29} \mathrm{Si}$ NMR spectrum also supported structural regularity. This polymer having the double-strand structure was found so stable as not to undergo depolymerization in solution. The present isolation of the crystalline polycondensate contributes to the understanding of the structure-property relationship of PPSQ.

\section{REFERENCES}

1. A. Ladenburg, Ber., 6, 379 (1873).

2. J. Meads and F. S. Kipping, J. Chem. Soc., 105, (1914).

3. K. Larsson, Arkiv Kemi, 16, 209 (1960).

4. K. Larsson and C. Grunwall, Arkiv Kemi., 17, 529 (1961).

5. J. F. Brown, Jr., L. H. Vogt, Jr., and P. I. Prescott, J. Am. Chem. Soc., 86, 1120 (1963).

6. J. F. Brown, J. Polym. Sci. Part C., 1, 83 (1963).

7. J. F. Brown, Jr. and L. H. Vogt, J. Am. Chem. Soc., 87, 4313 (1965).

8. M. M. Sprung and F. O. Guenther. J. Polym. Sci., 28, 17 (1958).

9. J. S. Hrkach and K. Matyjaszewski, J. Polym. Sci., A, Polym. Chem., 33, 285 (1995).

10. K. J. Mcneil, J. A. Dicaprio, D. A. Walsh, and R. F. Pratt, J. Am. Chem. Soc., 102, 1859 (1980).

11. T. Takiguchi, R. Fujikawa, Y. Yamamoto, and M. Ueda, Nippon Kagaku Kaishi, 108 (1974).

12. E. C. Lee and Y. Kimura, Polym. J., 29, 678 (1997).

13. H. Nishida, H. Yamane, Y. Kimura, and T. Kitao, Kobunshi Ronbunshu, 53, 193 (1996).

14. H. Ban, A. Tanaka, Y. Kawai, and S. Imamura, Polymer, 31, 564 (1990).

15. M. Smaihi, T. Jermoumi, and J. Marignan, Chem. Mater., 7, 2298 (1995).

16. P. Xie, Y. Wan, B. Zhou, J. Hou, D. Dai, Z. Li, D. Liu, and R. Zhang, Macromol. Chem. Phys., 197, 745 (1996).

17. T. Takiguchi, J. Am. Chem. Soc., 81, 2359 (1959).

18. B. L. Tyler, J. Am. Chem. Soc., 77, 770 (1955).

19. K. A. Andrianov, G. L. Slonimsky, A. A. Zhdanov, D. Ya. Tsvankin, V. Yu. Levin, V. S. Papkov, Yu. P. Kvachev, and E. M. Belavtseva, J. Polym. Sci., 14, 1205 (1976).

20. M. Konda, E. C. Lee, and Y. Kimura, unpublished data.

21. We have reported $59.6 \mathrm{MHz}{ }^{29} \mathrm{Si}$ NMR spectra of the TCP hydrolysates having different molecular weight. Here we reinvestigated the same spectra at a higher frequency of $99.3 \mathrm{MHz}$ by using a pure TMS in a cappilary tube as the in situ external reference. The chemical shift of the signals was found to be significantly different from the former value by $\delta-7.5 \mathrm{ppm}$ which had been measured with the signal of a sample of TMS in chloroform- $d_{1}$ as the machinary $0 \mathrm{ppm}$ (see ref 12 ).

22. J. Chojnowski, Spec. Publ. R. Soc. Chem., 166, 59 (1995). 